\title{
APOBEC3 inhibits DEAD-END function to regulate microRNA activity
}

\author{
Sara Ali ${ }^{1}$, Namrata Karki ${ }^{3}$, Chitralekha Bhattacharya ${ }^{1}$, Rui Zhu' ${ }^{1}$, Donna A MacDuff ${ }^{2}$, Mark D Stenglein², \\ April J Schumacher ${ }^{2}$, Zachary L Demorest ${ }^{2}$, Reuben S Harris², Angabin Matin ${ }^{1 *}$ and Sita Aggarwal ${ }^{3^{*}}$
}

\begin{abstract}
The RNA binding protein DEAD-END (DND1) is one of the few proteins known to regulate microRNA (miRNA) activity at the level of miRNA-mRNA interaction. DND1 blocks miRNA interaction with the $3^{\prime}$-untranslated region (3'-UTR) of specific mRNAs and restores protein expression. Previously, we showed that the DNA cytosine deaminase, APOBEC3 (apolipoprotein B mRNA-editing enzyme, catalytic polypeptide like 3), interacts with DND1. APOBEC3 has been primarily studied for its role in restricting and inactivating retroviruses and retroelements. In this report, we examine the significance of DND1-APOBEC3 interaction. We found that while human DND1 inhibits miRNA-mediated inhibition of P27, human APOBEC3G is able to counteract this repression and restore miRNA activity. APOBEC3G, by itself, does not affect the 3'-UTR of P27. We found that APOBEC3G also blocks DND1 function to restore miR-372 and miR-206 inhibition through the 3'-UTRs of LATS2 and CX43, respectively. In corollary experiments, we tested whether DND1 affects the viral restriction function or mutator activity of APOBEC3. We found that DND1 does not affect APOBEC3 inhibition of infectivity of exogenous retrovirus HIV ( $\triangle \mathrm{Vif}$ ) or retrotransposition of MusD. In addition, examination of Ter/Ter;Apobec3-/- mice, lead us to conclude that DND1 does not regulate the mutator activity of APOBEC3 in germ cells. In summary, our results show that APOBEC3 is able to modulate DND1 function to regulate miRNA mediated translational regulation in cells but DND1 does not affect known APOBEC3 function.
\end{abstract}

Keywords: DND1, APOBEC3G, APOBEC3, microRNA, P27

\section{Background}

The RNA binding protein DEAD-END (DND1) is essential for germ cell viability [1,2]. When Dnd1 is functionally inactivated, as in the Ter mutant mouse strain, this results in death of germ cells, sterility [2], and in some cases development of testicular germ cell tumors [2,3].

DND1 encodes canonical RNA recognition motifs $[1,4]$ through which it interacts with the 3'-UTRs of mRNAs. For example, DND1 inhibits miR-221 function from the 3'-UTR of P27 resulting in increased P27 protein expression $[4,5]$. Two U-rich DND1 binding sites have been mapped adjacent to two miR-221 binding sites in the 3'UTR of P27 [4]. DND1 has also been shown to inhibit miR-372 from the 3'-UTRs of LATS2 (serine/threonineprotein kinase, large tumor suppressor, homolog 2) and

\footnotetext{
*Correspondence: amatin@mdanderson.org; sita.aggarwal@pbrc.edu

'Department of Genetics, University of Texas, MD Anderson Cancer Center,

1515 Holcombe Blvd, Houston, TX 77030, USA

${ }^{3}$ Pennington Biomedical Research Center, 6400 Perkins Road, Baton Rouge, LA 70808, USA

Full list of author information is available at the end of the article
}

inhibit miR-1 and miR-206 from the 3'-UTRs of CX43 (connexin-43) [4]. However, DND1 binding sites have not been mapped within the 3'-UTRs of LATS2 or CX43.

miRNA association with mRNA usually results in translation inhibition or degradation of mRNA. It is thought that DND1 binds to mRNA and prevents miRNAs and miRISC (miRNA-induced silencing complexes) from binding. miRISCs are composed of ribonucleoproteins that assemble with the miRNA and mediate either translational repression or degradation of mRNA [6-8]. Alternately, DND1 may bind and sequester mRNAs away from miRNA access.

Although DND1 was initially identified for its role in germ cells and germ cell tumors, emerging evidence indicates a wider role for DND1 in mammalian tissues, especially in cancers. For example, over-expression of DND1 is detected in some histological sub-types of human testicular cancers, leukemia, lung and ovarian cancers (ONCOMINE and NCBI Geo Profiles). A recent study detected DND1 in human tongue squamous cell 
carcinoma (TSCC) and found that miR-24 directly targets DND1 mRNA [9]. Up-regulation of miR-24 decreased DND1 expression resulting in lower P27 levels and increased proliferation and reduced apoptosis in TSCC cells. Another study showed that Ras transformed keratinocytes down regulate DND1 which results in increased miR-21 mediated inhibition of MSH2 [10].

Work in our laboratory and others show that DND1 interacts with a broad range of mRNA targets $[11,12]$. The targets include transcripts encoding cell cycle regulators (P27, TP53, LATS2), pluripotency factors (OCT4, $S O X 2, N A N O G)$ and pro- and anti-apoptotic factors $(B A X$ and $B C L X)$. Expression of these genes is required at specific developmental stages in germ cells such as during active proliferation or quiescence. Because DND1 interacts with a range of mRNAs, this raises the question as to the factors which might serve to modulate DND1 interaction with physiologically appropriate targets. Therefore one important goal is to determine how DND1 function is regulated in cells.

In a related study, we found that DND1 interacts with APOBEC3 [13]. We showed that mouse DND1 immunoprecipitated with mouse APOBEC3 in mammalian cells, including in germ cells. In addition, fluorescent tagged DND1 and APOBEC3 co-localized at peri-nuclear regions in mammalian cells.

One well-studied function of mouse Apobec3 and its human counterpart, APOBEC3G (apolipoprotein B mRNA-editing enzyme, catalytic polypeptide-like 3G, A3G) is contributing to innate immunity through retrovirus and retrotransposon restriction [14-16]. Restriction occurs through a well-established cDNA cytosine deamination mechanism and by a less well-characterized deamination-independent mechanism [17-20]. Human APOBEC3G and mouse APOBEC3, each possess two zinc-binding motifs $[21,22]$. The active domain is responsible for deaminase activity and the pseudo-active domain contributes most of the RNA/ssDNA binding affinity [23-27]. Although both human and mouse proteins have such a division of labor, the domain organization is opposite with $\mathrm{N}$-terminal of mouse APOBEC3 and the C-terminal domain of human APOBEC3G active for deamination [28-30].

Other studies indicate that overexpression of specific members of the human APOBEC3 family (such as the single cytidine deaminase domain containing, APOBEC3A) can hypermutate the cellular genome or mitochondrial DNA [31,32]. Thus APOBEC3 family members are potentially powerful mutators [33] and very likely cells possess mechanisms to keep the latent deleterious activity of APOBEC3 in check. One way that cells protect their genomes from APOBEC3 is that mouse APOBEC3 and most human APOBEC3 proteins are localized to the cytoplasm $[14,29,34]$.
In this report, we examined the significance of APOBEC3 interaction with DND1. Our results show that APOBEC3 can oppose DND1 function to restore miRNA-mediated inhibition of translation. We therefore propose that interaction of APOBEC3 with DND1 may be one way in which DND1 activity is regulated in cells.

\section{Methods}

\section{Transient transfections}

Human DND1 with HA tag in C-terminus was cloned into pCDNA3.1 nV5-DEST (Invitrogen) expression vector (DND1-HA). Human APOBEC3G with myc tag in C-terminus was cloned into pcDNA3.1(+)(APOBEC3Gmyc). Transient transfections were performed using the $293 \mathrm{~T}$ cell line as this cell line has previously been used for testing DND1 function [4] and the results using $293 \mathrm{~T}$ were similar to that using other cell lines such as MCF-7 and Tera1. On advantage is that $293 \mathrm{~T}$ cells take up transfected DNA efficiently to give reproducible results. 293 T cells were cultured in DMEM supplemented with $10 \%$ fetal bovine serum in $5 \% \mathrm{CO}_{2}$ at $37^{\circ} \mathrm{C}$. The cells were transiently transfected using SuperFect transfection reagent (QIAGEN) with 1 ng pGL3-P27-3'UTR [4] together with constructs encoding miRVec-221 (50 ng), DND1-HA (10 ng) and/or APOBEC3G-myc (1 ng to $25 \mathrm{ng}$ range). Lac $Z$ expression constructs (4 ng) were co-transfected into all cells. Equivalent amounts of DNA were introduced into all cells with pGEM DNA being used to equalize for DNA levels used for transfections. After $48 \mathrm{~h}$ the cells were washed and treated with cell culture lysis buffer (Promega). $5 \mathrm{uL}$ of the lysates were used for luciferin assays. All transfection experiments were performed in triplicates. Results shown are the mean and standard error from three independent experiments. Similar transfections also tested the effect of DND1, APOBEC3G and miR-372 (mirVec-372) on pGL3 3'UTR LATS2, and miR-206 (miR vec-206) on pGL3 Cx43 3'UTR and pGL3-control vector. Mutant P27 vectors used were pGL3 3'UTR min mut1 ( $\mathrm{m} 1$ or mut1, in which both DND1 binding sites are mutated) and pGL3-p27mut-3'-UTR (m3; in which both miRNA binding sites are mutated) [4].

\section{Statistical analysis}

Data are expressed as mean \pm standard deviation/or standard error. Statistical analyses were performed using GraphPad Prism (software version 5.0. VA). Differences were determined by Student's t test. A $P$ value of $<0.05$ was considered significant.

\section{Luciferase assays}

The assays were performed using Luciferase assay kit (Pomega) according to manufacturer's directions. $\beta$ galactosidase assay results were used to normalize the 
transfection efficiencies. $\beta$-galactosidase assays were done using beta-Glo assay kit (Promega) according to manufacturer's direction.

\section{Immunoblotting}

DND1-HA and APOBEC3G-myc expression in transfected cells was detected in cell lysates using anti-myc and anti-HA antibodies, as described [13].

\section{Viral infectivity and MusD transposition assays}

Single cycle infectivity assays for $H I V(\Delta V$ if $)$ were performed using $293 \mathrm{~T}$ cells as described in [28]. MusD transposition assay in HeLa cells were performed as described in [35]. Expression vectors encoding mouse DND1 and APOBEC3 have been described [13,36].

\section{Mouse crosses}

Ter/+ mice were intercrossed with Apobec3-/- mice. (Both copies of wild-type DND1 are functionally inactivated in Ter/Ter mice.) The F1 mice were selected by genotyping and Ter/+;Apobec3+/- mice were intercrossed to generate Ter/Ter;Apobec3-/- mice [2,37]. The F2 mice were genotyped and the testes of double homozygote mice were examined for presence of germ cells. All mice were housed in the standard mouse Plexiglas cages in a room maintained at constant temperature and humidity under a $12 \mathrm{~h}$ light and darkness cycle. Animals were fed irradiated pelleted chow and water ad libitum. The experimental protocol was reviewed and approved by the Institutional Animal Care and Use Committee at MD Anderson Cancer Center.

\section{Results}

\section{APOBEC3 inhibits DND1 function}

Kedde et. al. [4] showed that DND1 blocks miR-221 function from the 3'-UTR of P27 mRNA. Also, a report by Huang et. al. [38] found that human APOBEC3G can inhibit miRNA function from mRNAs and this indicated a novel role of APOBEC3G in regulating miRNA activity and protein translation. Therefore, we asked whether APOBEC3 affects this function of DND1 and tested the effect of human APOBEC3G on DND1. Because 3'UTR of human $P 27$ has been characterized to contain two DND1 binding sites flanked by miR-221 binding sites (Figure 1a) [4], constructs encoding human P27 were predominantly used in these assays, together with human DND1 and APOBEC3G. We used the reporter construct, pGL3-P27-3'UTR [4], in which the 3'-UTR of human $P 27$ has been cloned downstream to luciferase reporter gene (Figure 1a). pGL3-P27-3'UTR was cotransfected into $293 \mathrm{~T}$ cells together with expression vectors encoding miR-221, HA-tagged DND1 (HA-tag in C-terminus of DND1) and myc-tagged APOBEC3G (myc-tag in C-terminus of APOBEC3G). Luciferase assays were carried out to monitor the effect of APOBEC3G and DND1 on miR-221 activity on P27 3'-UTR.

As expected, we found that miR-221 inhibits pGL3P27-3'UTR luciferase activity $(P=0.005)$. DND1 counteracts the effect of miR-221 to restore luciferase activity $(P=0.01) \quad$ (Figure 1b). However, when we introduce APOBEC3G together with DND1, we found that presence of APOBEC3G opposes DND1 repression of miR-221 $(P=0.009)$ (Figure $1 c)$. Thus APOBEC3G restores miR221 inhibition of pGL3-p27-3'UTR luciferase activity.

APOBEC3G had a similar effect of blocking DND1 function to restore miR-206 inhibition from $C X 43$ (connexin-43; pGL3 Cx43 3'UTR) $(P=0.02)$ and to restore miR-372 inhibition from the $3^{\prime}$-UTR of LATS2 (pGL3 3'UTR LATS2) $(P=0.001)$ (Figure 1d and 1e). In these experiments, APOBEC3G restored the miRmediated inhibition of luciferase activity but did not further increase inhibition. We conclude therefore, that APOBEC3G may have a general function in blocking DND1 activity. We verified that transfected plasmids encoding HA-tagged DND1 and myc-tagged APOBEC3G were expressed in the $293 \mathrm{~T}$ cells (Figure 11). We also detected expression of endogenous DND1, but not APOBEC3G, in 293 T cells (Figure 1m).

Control experiments demonstrated no significant effect of either DND1 or APOBEC3G alone (Figure 1f-h) or DND1 plus APOBEC3G on luciferase activity of pGL3P27-3'-UTR, pGL3-Cx43-3'UTR or pGL3-3'UTR-LATS2 (Figure 1i-k).

In further control experiments, we used P27 3'-UTR constructs in which the DND1 or miRNA binding sites were mutated [4] (Figure 2b and c). When both DND1 binding sites on pGL3-P27-3'-UTR were inactivated (m1-luc), miR-221 was able to suppress luciferase activity but DND1 was unable to rescue miR-221 inhibition $(P=0.3)$ and APOBEC3G was not able to restore miR221 inhibition $(P=0.3)$ (Figure $2 b)$. This suggests that APOBEC3G likely functions, at least partially, through its interaction with DND1 or through DND1 binding sites on $P 27$. Use of mutant P27 3'-UTR constructs in which the miRNA binding sites were mutated (m3-luc) [4] (Figure 2c) showed, as expected, no functional inhibitory effect of mir-221 or any further effects by DND1 or APOBEC3G.

Next we tested the effect of higher APOBEC3G concentrations on normal and mutant luciferase expression constructs (Figure 2d-i). Levels that we normally use in our reporter assays (10 ng), had no effect on pGL3-P273 '-UTR, luc-m1, luc-m3 expression (Figure 2d-f). However, higher levels of APOBEC3G (25 ng) slightly inhibited pGL3-P27-3'-UTR $(P=0.023)$ expression and on the other hand, slightly enhanced luc-m1 (both DND1 binding sites mutated) $(P=0.04)$ and luc-m3 expression (both miR-221 binding sites mutated) $(P=0.01)$ (Figure 2d-f). We don't fully understand why higher 


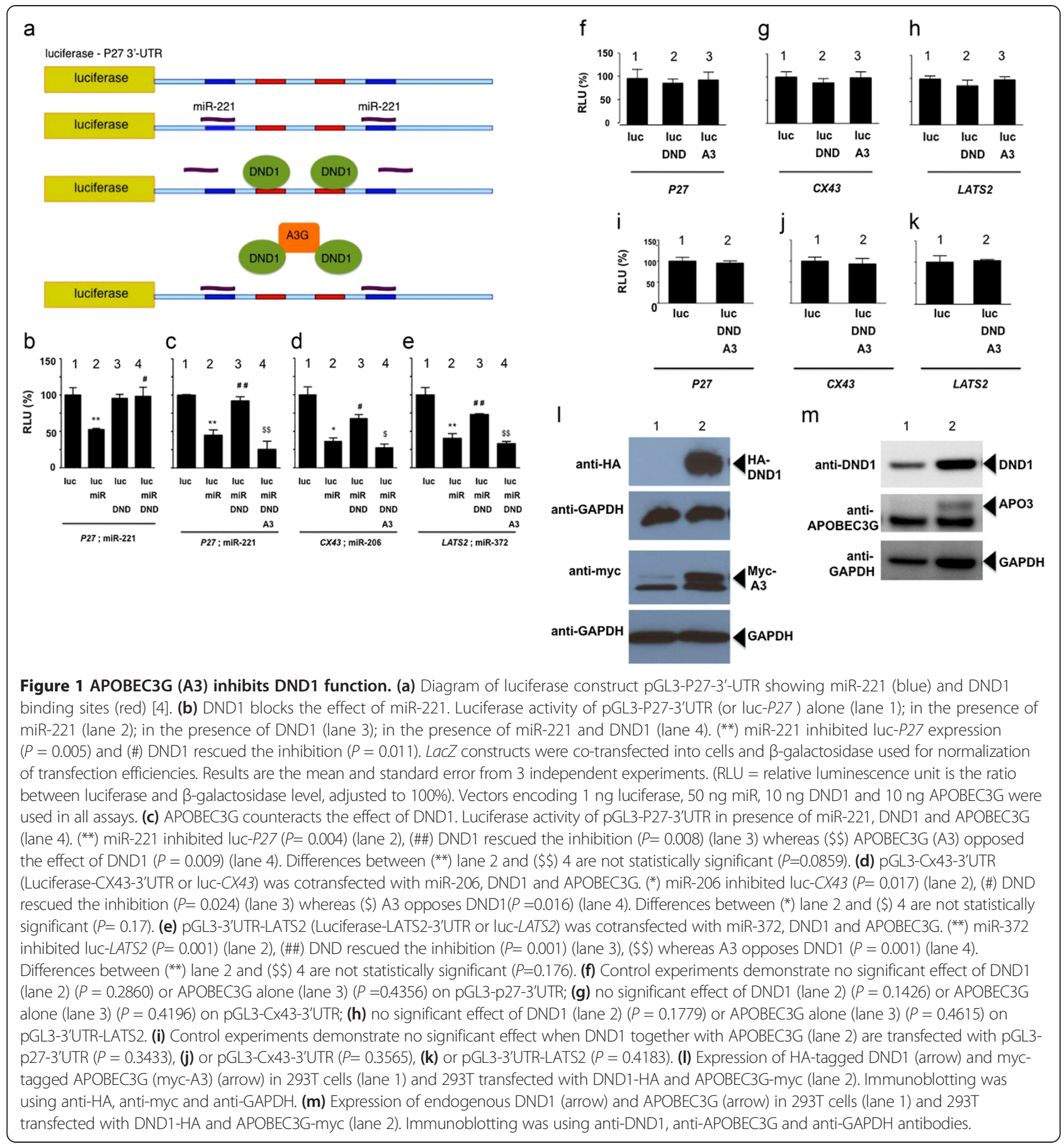

levels of APOBEC3G affect luc-P27, $\mathrm{m} 1$ and $\mathrm{m} 3$ activity. One possibility is that at higher concentrations, APOBEC3G may have additional functions such as ability to interact with the $3^{\prime}$-UTRs to inhibit translation and luciferase activity (of wild-type P27) or promote translation (from mutant $\mathrm{m} 1$ and $\mathrm{m} 3$ ).

Higher APOBEC3G, when present together with miR221, also further inhibited pGL3-P27-3'-UTR (or lucP27) $(P=0.0488)$, luc-CX43 $(P=0.0385)$ and luc-LATS2
$(P=0.013)$ (Figure 2g-i). One explanation for this is that because $293 \mathrm{~T}$ cells express endogenous DND1 (Figure $1 \mathrm{~m}$ ), higher concentrations of APOBEC3G are able to more effectively block the effect of both endogenous and transfected DND1 to restore miRNAmediated inhibition. Also, interestingly, an earlier study showed that APOBEC3G can, by itself, inhibit miRNA activity, and these studies utilized higher, microgram levels of APOBEC3G [38]. 




We also tested the direct effect of APOBEC3G on pGL3-control vector. Increasing levels of APOBEC3G did not affect the luciferase activity from pGL3-control vector implying that APOBEC3G did not affect translation of luciferase (Figure 2j). The pGL3 vector does not have an extensive $3^{\prime}$-UTR with miRNA or DND1 binding sites unlike the $3^{\prime}$-UTRs of P27, CX43 or LATS2, and therefore is not affected by higher levels of APOBEC3G.

In summary, these experiments lead us to conclude that APOBEC3G is able to block DND1 function and restore miRNA-mediated translation repression. APOBEC3G does not directly affect the 3'-UTRs of P27, CX43 or LATS2 or miRNA interaction with mRNAs. However, higher levels of APOBEC3G appear to have additional independent functions on the 3'-UTRs of genes.

\section{DND1 does not affect APOBEC3 function}

The above results show that APOBEC3G can block DND1 function to regulate miRNA activity. However, mouse APOBEC3 and human APOBEC3G have been widely studied for their role in inhibiting viral infectivity [14-16]. Therefore, we tested whether DND1 affects APOBEC3 function of inhibiting retroviral infectivity $[28,39]$. Single cycle infectivity assay shows that, as expected, mouse APOBEC3 severely reduces $\mathrm{HIV}(\Delta \mathrm{Vif})$ viral infectivity in $293 \mathrm{~T}$ cells (Figure 3a, lane 3). However, presence of mouse DND1 together with APOBEC3 did not change ability of APOBEC3 to reduce $\mathrm{HIV}(\Delta \mathrm{Vif})$ infectivity (Figure 3a, lane 4). DND1 alone also had no effect on $\operatorname{HIV}(\Delta$ Vif) viral infectivity (Figure 3a, lane 2 ). Empty vector was used to equalize the amount of construct used in each assay lane $[28,39]$. 


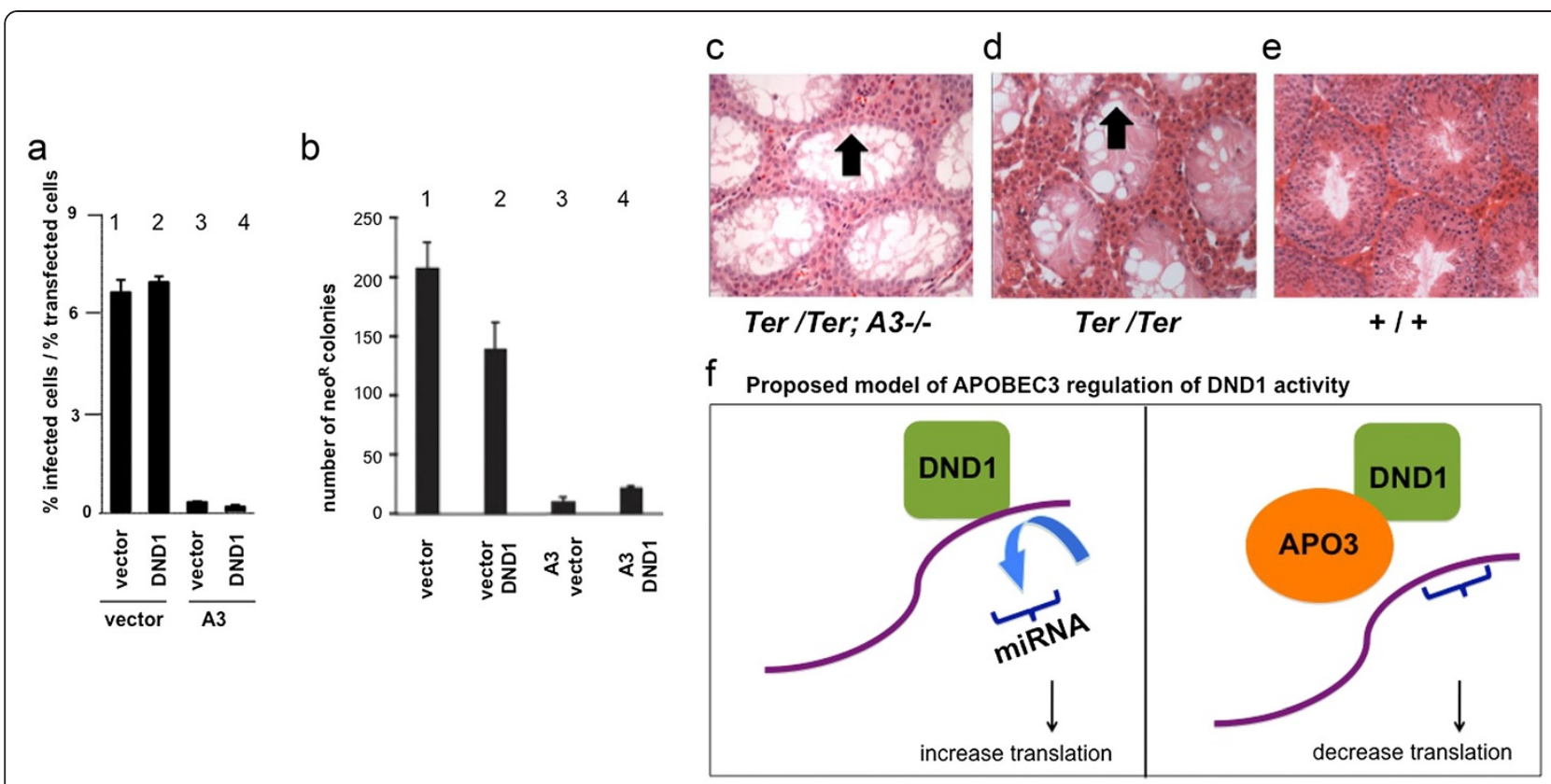

Figure 3 DND1 does not affect APOBEC3 function. (a) DND1 does not affect APOBEC3 antiretroviral activity. Infectivity of HIV-GFP produced in the presence of control vector (vector) (lane 1) or vector encoding mouse DND1 (lane 2) $(P=0.2082)$. Infectivity of HIV-GFP produced in the presence of mouse APOBEC3 (A3) (lane 3) and APOBEC3 plus DND1 (lane 4) ( $P=0.1464)$. Empty vector was used to equalize the amount of construct used in each assay lane. Results from two independent experiments were averaged. Error bars indicate the difference in infectivity observed between the two experiments. (b) DND1 does not affect MusD restriction by APOBEC3. Effect of control vector (lane 1), mouse DND1 (lane 2), mouse APOBEC3 (lane 3), both mouse DND1 and APOBEC3 (lane 4) on MusD retrotransposition, relative to the vector control. Transposition was monitored by the number of G418-resistant colonies. Results from two independent experiments were averaged. $P$ value comparing lanes 3 and 4 is 0.10980 . (c) Histology section through testes of double homozygous male Ter/Ter ;A3-/- (or Ter/Ter; Apobec3-/-), (d) Ter/Ter and (e) wild-type (+/+) mice. Arrow points to lumen in seminiferous tubules showing lack of germ cells persist in Ter/Ter; A3-/-testis similar to that in testis of Ter/Ter mice. Testes of Apobec3-/- (A3-/-) mice have normal wild-type germ cell histology similar to +/+ (not shown). (f) Proposed model how APOBEC3G regulates DND1 function. DND1 blocks miRNA activity (left panel). We observe decreased protein translation (as measured by luciferase activity) in the presence of APOBECG3 suggesting that APOBEC3G blocks DND1 function. When DND1 binding sites on P27-3'-UTR are inactivated, both DND1 and APOBEC3G fail to affect luciferase activity. This suggests that APOBEC3G functions through DND1, may be by removing or sequestering DND1 to restore miRNA access to the $3^{\prime}-U T R$ of $P 27$ (right panel).

In a second set of experiments, we tested whether DND1 affects the ability of APOBEC3 to inhibit MusD retrotransposition [35]. APOBEC3, by itself, drastically inhibits MusD retrotransposition (as indicated by decrease in neo $^{\mathrm{R}}$ colonies by Apo3 + vector) (Figure 3b, lane 3). However, DND1, when combined with APOBEC3, did not significantly affect APOBEC3 function of inhibiting MusD retrotransposition (A3 + DND1, lane 4). DND1, by itself, also had no effect on MusD retrotransposition (vector + DND1, lane 2). The results from these two experiments indicate that DND1 does not modulate the viral restriction function of APOBEC3.

To further explore the functional relationship between Dnd1 and Apobec3, we asked whether the germ cell phenotype of mice lacking wild-type Dnd1 (Ter mutant mice) is dependent on Apobec3. It is known that APOBEC3 family members are potentially powerful mutators [31-33]. We reasoned that interaction of DND1 with APOBEC3 in germ cells may be one mechanism to keep the latent deleterious activity of
APOBEC3 in check. Thus it is formally possible that depletion of germ cells in mice lacking normal Dnd1 (as observed in Ter mutant mice) is due to uncontrolled Apobec3 genomic mutating activity. We therefore tested whether removing Apobec3 from mice in which Dnd1 is inactivated, would restore normal germ cells. Mice lacking wild-type Dnd1 (in Ter mice) have no germ cells [2] but mice lacking Apobec3 are normal and fertile [37]. Thus, we generated double mutant mice lacking both wild-type Dnd1 and Apobec3 (Ter/Ter;Apobec3-/- or Ter/Ter;A3-/-) mice. However, examination of newborn or adult testes of Ter/Ter;A3-I- mice indicated that they have no germ cells and are sterile (Figure $3 \mathrm{c}$ ) and are indistinguishable from Ter/Ter;A3+/+ animals (Figure 3d). Thus this genetic assay suggests that DND1 does not regulate the mutator function of APOBEC3 in germ cells and germ cell loss.

in Ter/Ter mice is likely not due to unregulated activity of APOBEC3. Together, results from the restriction assays and the genetic crosses are consistent with the 
idea that DND1 does not modulate mouse APOBEC3 activity.

\section{Discussion}

Our results demonstrate that APOBEC3G is able to block DND1 function and restore miRNA mediated inhibition of translational repression. This function of APOBEC3G appears to apply to multiple mRNA targets of DND1 as APOBEC3G has a similar effect on P27, LATS2 and CX43. On the other hand, DND1 does not appear to affect the viral restriction function of APOBEC3. In addition, our genetic crosses suggest that DND1 interaction with APOBEC3 does not regulate the mutator function of APOBEC3 in germ cells.

The mechanism of how APOBEC3G blocks DND1 remains to be determined. At least, three possible mechanisms can be proposed. The first possibility is that APOBEC3G may bind to DND1 and sequester it away from mRNAs and miRNAs (Figure 3f). In support of this, we have observed that mouse APOBEC3 and DND1 co-immunoprecipitate and co-localize in cells [13].

The second possibility is that APOBEC3G may bind mRNAs (maybe together with DND1) and subsequently interact with components of the miRISC to activate translation repression or interact with translation initiation factors to inhibit them. Indeed, mass spectrometric analysis show that a large number of cellular RNAbinding proteins associate with APOBEC3G [40-42]. Some of these are known components of the miRISC such as ARGONAUTE 1(Ago1), ARGONAUTE 2 (Ago2), GW182, MOV10, YB-1, DCP1A and RCK/P54, and are involved in post-transcriptional silencing of gene expression [38,41-43]. These interactions of APOBEC3G with RNA binding proteins were found to be either direct protein-protein interactions or mediated by RNA. In addition, confocal microscopy experiments showed that APOBEC3G co-localized with many of the miRISC RNA-binding proteins to mRNA processing, P-bodies $[40,41]$. Thus it is conceivable that interaction of APOBEC3 with specific miRISC proteins may override the effect of DND1 to enhance miRNA activity.

The third possibility is that the cytidine deaminase activity of APOBEC3 may allow it to edit the 3 '-UTR sequences of P27, LATS2 and CX43 to inhibit DND1 binding. Interestingly, indirect support of this hypothesis comes from recent reports by a number of groups that analysed deep sequencing data and found greater than expected incidence of editing present in the mammalian transcriptome $[44,45]$. As to which of the three possible mechanisms apply to APOBEC3G blocking DND1 function is currently under investigation.

APOBEC3 proteins have been studied as factors that restrict viruses and retrotransposons. However, we found that DND1 has no effect on the viral restriction function of APOBEC3. APOBEC3G can bind both cellular RNAs and RNA binding proteins $[27,40,41]$ and the RNA binding activity of the $\mathrm{N}$-terminal cytidine deaminase domain of APOBEC3G is essential for viral restriction [23,24]. The significance of APOBEC3G interactions with cellular proteins and RNAs is not clear. However, during viral infection of cells, such as HIV-1 (Vif) infection of T lymphocytes, APOBEC3G gains access to viral particles through a ribonucleoprotein interaction and thus APOBEC3 binding to RNA is a critical for antiviral function [23]. The incorporation of APOBEC3G into new viral particles allows it to be released into infected cells where APOBEC3G can deaminate the replicating viral cDNAs to effect reduction of viral infectivity. Interestingly, it has been shown that other P-body proteins, such as MOV10 [46-48] are also involved in reducing the infectivity of exogenous retroviruses and retrotransposons. Thus, P-body protein components such as MOV10, and as we report here, APOBEC3G, participate both in miRNA silencing and viral restriction processes.

An earlier study on APOBEC3G function showed that APOBEC3G can, by itself, inhibit miRNA activity [38]. The previous study used luciferase constructs encoding only miRNA binding sites or encoding multiple miRNA binding sites in tandem. In contrast, our assays used the 3'-UTR of endogenous genes to test APOBEC3G activity and we also tested how APOBEC3G blocks DND1 function on these 3'-UTRs. Moreover, the amount of APOBEC3G expression vectors transfected into cells were considerably higher in the study [38] and we also found that higher APOBEC3G levels may have alternate effects on the 3'-UTRs and miRNA function. Another possibility is that APOBEC3G may have different effects on different miRNAs and transcripts, inhibiting some miRNAs while activating others.

One caveat of our studies is that the miRNA studies were performed using human DND1 and APOBEC3G whereas the infectivity assays and genetic studies were performed using mouse factors. We did this because human DND1 function was previously characterized using human P27 3'-UTR and DND1 binding sites have been mapped in the 3'UTR of P27 [4]. Humans encode multiple members of the APOBEC3 family proteins (APOBEC3A, 3B, 3C, etc.) [21]. We selected human APOBEC3G for our miRNA studies which is functionally most closely related to mouse APOBEC3. The role of the other human APOBEC3 factors in inhibiting DND1 activity remains to be determined.

APOBEC3 proteins are expressed in germ cells $[13,21,49]$. But although APOBEC3 proteins inhibit retrotransposition $[17,50,51]$, to date, this has not been demonstrated in germ cells. In fact, Apobec 3 null mice are normal and fertile [37]. We found that double null Ter/Ter;Apobec3-/- mice have similar phenotype as 
Ter/Ter mice and lack of Apobec3 does not rescue the Ter/Ter (Dnd1-/-) phenotype to restore germ cells. This supports the idea that DND1 does not regulate the mutator activity of APOBEC3 in germ cells, and deregulated APOBEC3 mutator activity is not responsible for germ cell loss in Ter/Ter (Dnd1-/-) mice.

In summary, our studies focus on a novel aspect of APOBEC3 function in that we show APOBEC3G regulates DND1 function and in this way affects miRNA activity. Viral restriction and miRNA mediated gene silencing are evolutionarily related processes utilizing similar protein complexes, which localizes to cytoplasmic RNA granules. Our data provides compelling evidence that APOBEC3G may be involved in both these processes.

\section{Conclusion}

We present our novel finding that RNA binding protein DEAD END (DND1) blocks miRNA function to permit translation, and APOBEC3G (apolipoprotein B mRNAediting enzyme, catalytic polypeptide like 3 ) antagonizes DND1 to reduce translation. Not much is known about how microRNA target interactions are regulated by RNA binding proteins, thus our result advances an area of importance to help understand how microRNA activity can be modulated in response to signals.

\section{Abbreviations \\ 3'-UTR: 3'-untranslated region; Mouse APOBEC3 and human \\ APOBEC3G: Apolipoprotein B mRNA-editing enzyme, catalytic polypeptide like 3; LATS2: Serine/threonine-protein kinase, large tumor suppressor, homolog 2; CX43: Connexin-43; miRNA: microRNA; mRNA: Messenger RNA; P27, LATS2: Human genes and transcripts are in italics and capitals; P27, LATS2, APOBEC3G: Human and mouse proteins are in capitals; Apobec3: Mouse genes and transcripts are in italics and lowercase.}

\section{Competing interests}

The authors declare that they have no competing interests.

\section{Authors' contributions}

AM and SA conceived and designed the experiments, performed analysis and interpretation of data and wrote the manuscript. NK performed the transfection assays. CB carried out the genetic crosses and mouse histology. RZ, S Ali and SA performed the experiments. DAM, MDS, AJS, ZLD and RH designed, performed and analyzed viral infectivity and MusD transposition assay data. All authors read and approved the final manuscript.

\section{Acknowledgements}

This project was funded by Texas ARP. We thank Q. Wang, M. Zhang, K. Luo, and G. Seawood for technical assistance. We thank R. Agami for the generous gift of the luciferase and mirVec constructs. We thank R. Harris for the human APOBEC3G-myc construct and M.S. Neuberger and C. Rada for Apobec3-/- mice.

\section{Author details}

'Department of Genetics, University of Texas, MD Anderson Cancer Center, 1515 Holcombe Blvd, Houston, TX 77030, USA. ²Department of Biochemistry, Molecular Biology, and Biophysics, University of Minnesota, 321 Church Street SE, Minneapolis, MN 55455, USA. ${ }^{3}$ Pennington Biomedical Research Center, 6400 Perkins Road, Baton Rouge, LA 70808, USA.

Received: 11 January 2013 Accepted: 9 July 2013

Published: 26 July 2013

\section{References}

1. Weidinger G, Stebler J, Slanchev K, Dumstrei K, Wise C, Lovell-Badge R, Thisse C, Thisse B, Raz E: Dead end, a novel vertebrate germ plasm component, is required for zebrafish primordial germ cell migration and survival. Curr Biol 2003, 13(16):1429-1434.

2. Youngren KK, Coveney D, Peng X, Bhattacharya C, Schmidt LS, Nickerson ML, Lamb BT, Deng JM, Behringer RR, Capel B, et al: The Ter mutation in the dead end gene causes germ cell loss and testicular germ cell tumours. Nat 2005, 435:360-364.

3. Noguchi T, Noguchi M: A recessive mutation (ter) causing germ cell deficiency and a high incidence of congenital testicular teratomas in 129/Sv-ter mice. J Natl Cancer Inst 1985, 75(2):385-392.

4. Kedde M, Strasser MJ, Boldajipour B, Vrielink JA, Slanchev K, le Sage C, Nagel $\mathrm{R}$, Voorhoeve PM, van Duijse J, Orom UA, et al: RNA-binding protein Dnd1 inhibits microRNA access to target mRNA. Cell 2007, 131:1273-1286.

5. Ketting RF: A dead end for microRNAs. Cell 2007, 131:1226-1227.

6. Bushati N, Cohen SM: microRNA functions. Ann Rev Cell Dev Biol 2007, 23:175-205.

7. Peters L, Meister G: Argonaute poteins: mediators of RNA silencing. Mol Cell 2007, 26:611-623.

8. Filipowicz W, Bhattacharya SN, Sonenberg N: Mechanisms of posttranscriptional regulation by microRNAs: are the answers in sight? Nat Rev Genet 2008, 9:102-114.

9. Liu X, Wang A, Heidbreder CE, Jiang L, Yu J, Kolokythas A, Huang L, Dai Y, Zhou X: MicroRNA-24 targeting RNA-binding protein DND1 in tongue squamous cell carcinoma. FEBS Lett 2010, 584:4115-4120.

10. Bhandari A, Gordon W, Dizon D, Hopkin AS, Gordon E, Yu Z, Andersen B: The Grainyhead transcription factor Grhl3/Get1 suppresses miR-21 expression and tumorigenesis in skin: modulation of the miR-21 target MSH2 by RNA-binding protein DND1. Oncogene 2012. doi:10.1038/ onc.2012.168:1-11.

11. Cook MS, Munger SC, Nadeau JH, Capel B: Regulation of male germ cell cycle arrest and differentiation by DND1 is modulated by genetic background. Dev 2011, 138:23-32.

12. Zhu R, lacovino M, Mahen E, Kyba M, Matin A: Transcripts that associate with the RNA binding protein, DEAD-END (DND1), in embryonic stem (ES) cells. BMC Mol Biol 2011, 12(1):37.

13. Bhattacharya C, Aggarwal S, Kumar M, Ali A, Matin A: Mouse apolipoprotein B editing complex 3 (APOBEC3) is expressed in germ cells and interacts with dead-end (DND1). PLoS One 2008, 3(5):e2315.

14. Mangeat B, Turelli P, Caron G, Friedli M, Perrin L, Trono D: Broad antiretroviral defence by human APOBEC3G through lethal editing of nascent reverse transcripts. Nat 2003, 424:99-103.

15. Shindo K, Takaori-Kondo A, Kobayashi M, Abudu A, Fukunaga K, Uchiyama $\mathrm{T}$ : The enzymatic activity of CEM15/Apobec-3G is essential for the regulation of the infectivity of HIV-1 virion but not a sole determinant of its antiviral activity. J Biol Chem 2003, 278:44412-44416.

16. Wissing S, NLK G, Greene WC: HIV-1 Vif versus the APOBEC3 cytidine deaminases: an intracellular duel between pathogen and host restriction factors. Mol Aspects Med 2010, 31:383-397.

17. Esnault C, Heidmann O, Delebecque F, Dewannieux M, Ribet D, Hance AJ, Heidmann T, Schwartz O: APOBEC3G cytidine deaminase inhibits retrotransposition of endogenous retroviruses. Nat 2005, 433:430-433.

18. Muckenfuss $H$, Hamdorf M, Held U, Perkovic M, Löwer J, Cichutek K, Flory E, Schumann GG, Münk C: APOBEC3 proteins inhibit human LINE-1 retrotransposition. J Biol Chem 2006, 281:22161-22172.

19. Bishop KN, Holmes RK, Malim MH: Antiviral potency of APOBEC proteins does not correlate with cytidine deamination. J Virol 2006, 80:8450-8458.

20. Iwatani Y, Chan DS, Wang F, Maynard KS, Sugiura W, Gronenborn AM, Rouzina I, Williams MC, Musier-Forsyth K, Levin JG: Deaminase independent inhibition of HIV-1 reverse transcription by APOBEC3G. Nucleic Acids Res 2007, 35:7096-7108.

21. Jarmuz A, Chester A, Bayliss J, Gisbourne J, Dunham I, Scott J, Navaratnam $\mathrm{N}$ : An anthropoid-specific locus of orphan C to U RNA-editing enzymes on Chromosome 22. Genomics 2002, 79:285-296.

22. Conticello SG, Thomas CJ, Petersen-Mahrt SK, Neuberger MS: Evolution of the AID/APOBEC family of polynucleotide (deoxy)cytidine deaminases. Mol Biol Evol 2005, 22:367-377.

23. Friew YN, Boyko $V$, Hu WS, Pathak VK: Intracellular interactions between APOBEC3G, RNA, and HIV-1 Gag: APOBEC3G multimerization is dependent on its association with RNA. Retroviro/ 2009, 6:56. 
24. Gooch BD, Cullen BR: Functional domain organization of human APOBEC3G. Virol 2008, 379:118-124.

25. Iwatani Y, Takeuchi H, Strebel K, Levin JG: Biochemical activities of highly purified, catalytically active human APOBEC3G: correlation with antiviral effect. J Virol 2006, 80:5992-6002.

26. Li J, Potash MJ, Volsky DJ: Functional domains of APOBEC3G required for antiviral activity. J Cell Biochem 2004, 92:560-572.

27. Navarro F, Bollman B, Chen H, Konig R, Yu Q, Chiles K, Landau NR: Complementary function of the two catalytic domains of APOBEC3G. Virol 2005, 333:374-386.

28. Hache G, Liddament MT, Harris RS: The retroviral hypermutation specificity of APOBEC3F and APOBEC3G is governed by the C-terminal DNA cytosine deaminase domain. J Biol Chem 2005, 280:10920-10924.

29. Jónsson SR, Haché G, Stenglein MD, Fahrenkrug SC, Andrésdóttir V, Harris RS: Evolutionarily conserved and non-conserved retrovirus restriction activities of artiodactyl APOBEC3F proteins. Nucleic Acids Res 2006, 34:5683-5694

30. Hakata Y, Landau NR: Reversed functional organization of mouse and human APOBEC3 cytidine deaminase domains. J Biol Chem 2006, 281:36624-36631.

31. Suspène R, Aynaud M-M, Guétard D, Henry M, Eckhoff G, Marchio A, Pineau $P$, Dejean A, Vartanian J-P, Wain-Hobson S: Somatic hypermutation of human mitochondrial and nuclear DNA by APOBEC3 cytidine deaminases, a pathway for DNA catabolism. Proc Natl Acad Sci USA 2011, 17:2-7.

32. Landry S, Narvaiza I, Linfesty DC, Weitzman MD: APOBEC3A can activate the DNA damage response and cause cell-cycle arrest. EMBO Rep 2011, 12:444-450.

33. Petit $V$, Vartanian J-P, Wain-Hobson S: Powerful mutators lurking in the genome. Phil Trans R Soc B 2009, 364:705-715.

34. Stenglein MD, Harris RS: APOBEC3B and APOBEC3F inhibit L1 retrotransposition by a DNA deamination-independent mechanism. J Biol Chem 2006, 281:16837-16841.

35. Schumacher AJ, Hache G, MacDuff DA, Brown WL, Harris RS: The DNA deaminase activity of human APOBEC3G is required for Ty1, MusD, and human immunodeficiency virus type 1 restriction. J Virol 2008, 82:2652-2660.

36. Bhattacharya C, Aggarwal S, Zhu R, Kumar M, Zhao M, Meistrich ML, Matin $A$ : The mouse dead-end gene isoform alpha is necessary for germ cell and embryonic viability. Biochem Biophys Res Commun 2007, 355:194-199.

37. Mikl MC, Watt IN, Lu M, Reik W, Davies SL, Neuberger MS, Rada C: Mice deficient in APOBEC2 and APOBEC3. Mol Cell Biol 2005, 25:7270-7277.

38. Huang J, Liang Z, Yang B, Tian H, Ma J, Zhang H: Derepression of microRNA-mediated protein translation inhibition by apolipoprotein $B$ mRNA-editing enzyme catalytic polypeptide-like $3 G$ (APOBEC3G) and its family member. J Biol Chem 2007, 282:33632-33640.

39. Schumacher AJ, Nissley DV, Harris RS: APOBEC3G hypermutates genomic DNA and inhibits Ty1 retrotransposition in yeast. Proc Natl Acad Sci USA 2005, 102:9854-9859.

40. Kozak SL, Marin M, Rose KM, Bystrom C, Kabat D: The anti-HIV-1 editing enzyme APOBEC3G binds HIV-1 RNA and messenger RNAs that shuttle between polysomes and stress granules. J Biol Chem 2006, 281:29105-29119.

41. Gallois-Montbrun S, Kramer B, Swanson CM, Byers H, Lynham S, Ward M, Malim MH: Antiviral protein APOBEC3G localizes to ribonucleoprotein complexes found in P bodies and stress granules. J Virol 2007, 81:2165-2178.

42. Gallois-Montbrun S, Holmes RK, Swanson CM, Fernandez-Ocana M, Byers HL, Ward MA, Malim MH: Comparison of cellular ribonucleoprotein complexes associated with the APOBEC3F and APOBEC3G antiviral proteins. J Virol 2008, 11:5636-5642.

43. Wichroski MJ, Robb GB, Rana TM: Human retroviral host restriction factors APOBEC3G and APOBEC3F localize to mRNA processing bodies. PLOS Pathog 2006, 2:374-383.

44. Zaranek AW, Levanon EY, Zecharia T, Clegg T, Church GM: A survey of genomic traces reveals a common sequencing error, RNA editing, and DNA editing. PLOS Genet 2010, 6:e1000954.

45. Li M, Wang IX, Li Y, Bruzel A, Richards AL, Toung JM, Cheung VG: Widespread RNA and DNA sequence differences in the human transcriptome. Sci 2011, 333:53-58.

46. Nathans R, Chu C, Serquina AK, Lu C-C, Cao H, Rana TM: Cellular microRNA and $\mathrm{P}$ bodies modulate host-HIV-1 interactions. Mol Cell 2009, 34:696-709.
47. Chable-Bessia C, Meziane O, Latreille D, Triboulet R, Zamborlini A, Wagschal A, Jacquet J-M, Reynes J, Levy Y, Saib A, et al: Suppression of HIV-1 replication by microRNA effectors. Retrovirol 2009, 6:26.

48. Burdick R, Smith JL, Chaipan C, Friew Y, Chen J, Venkatachari NJ, DelviksFrankenberry KA, Hu W-S, Pathak VK: P body-associated protein Mov10 inhibits HIV-1 replication at multiple stages. J Virol 2010, 84:10241-10253.

49. Koning FA, Newman ENC, Kim E-Y, Kunstman KJ, Wolinsky SM, Malim MH: Defining APOBEC3 expression patterns in human tissues and hematopoietic cell subsets. J Virol 2009, 83:9474-9485.

50. Feinberg AP, Ohlsson R, Henikoff S: The epigenetic progenitor origin of human cancer. Nature Rev Genet 2006, 7:21-33.

51. Bogerd HP, Wiegand HL, Doehle BP, Lueders KK, Cullen BR: APOBEC3A and $A P O B E C 3 B$ are potent inhibitors of LTR-retrotransposon function in human cells. Nucleic Acids Res 2006, 34:89-95.

doi:10.1186/1471-2199-14-16

Cite this article as: Ali et al:: APOBEC3 inhibits DEAD-END function to regulate microRNA activity. BMC Molecular Biology 2013 14:16.

\section{Submit your next manuscript to BioMed Central and take full advantage of:}

- Convenient online submission

- Thorough peer review

- No space constraints or color figure charges

- Immediate publication on acceptance

- Inclusion in PubMed, CAS, Scopus and Google Scholar

- Research which is freely available for redistribution

Submit your manuscript at www.biomedcentral.com/submit
C BioMed Central 